\title{
IMPULSIVE INTERGO-DIFFERENTIAL EQUATIONS AND STABILITY OF MOVING INVARIANT MANIFOLDS*
}

\author{
GANI TR. STAMOV ${ }^{\dagger}$
}

\begin{abstract}
This paper study the stability of moving invariant manifolds of nonlinear impulsive integro-differential equations. The obtain results are based on the method of piecewise continuous Lyapunov's functions and the comparison principle.
\end{abstract}

Key words. Uncertain impulsive integro-differential system, moving invariant manifold, stability theory

AMS subject classifications. $34 \mathrm{~A} 37$

1. Preliminary notes. Impulsive integro-differential equations arise naturally from a wide variety of applications such as aircraft control, inspection process in operations research, drug administration, and threshold theory in biology. There has been a significant development in the theory of impulsive differential equations in the last years [1-3].

Now there also exist a well developed qualitative theory for impulsive integrodifferential equations $[7,8]$.

The efficient applications of impulsive integro-differential equations to mathematical simulation request the finding of criteria for stability of their solutions.

In this paper we use piecewise continuous Lyapunov's functions to study the stability of moving invariant manifolds for general class of uncertain impulsive integrodifferential equations. In the few publications dedicated to the subject of moving invariant manifold for differential equations without impulses, earlier works were done by $[5,6-7,10]$.

Our results are obtained by means of the comparison principle which permits us to reduce the study of impulsive integro-differential equations to the study of a scalar differential equation.

Let $\mathbb{R}^{n}$ be the $n$-dimensional Euclidean space with elements $x=\operatorname{col}\left(x_{1}, x_{2}, \ldots, x_{n}\right)$ and norm $|\cdot|, \mathbb{R}=(-\infty, \infty), \mathbb{R}_{+}=[0, \infty), S_{\rho}=\left\{x \in \mathbb{R}^{n},|x|=\rho\right\}, B_{\rho}=\left\{x \in \mathbb{R}^{n}\right.$ : $|x|<\rho\}, \rho>0$.

We shall consider the following system of uncertain impulsive integro-differential equations

$$
\left\{\begin{array}{l}
\dot{x}(t)=F(t, x(t),(T x)(t), \lambda), t \neq \tau_{k} \\
\Delta x\left(\tau_{k}\right)=I_{k}\left(x\left(\tau_{k}\right), \lambda\right), k=1,2, \ldots \\
x\left(t_{0}+0\right)=x_{0}, t_{0} \in \mathbb{R}_{+}
\end{array}\right.
$$

where
i) $F \in C\left(\mathbb{R}_{+} \times \mathbb{R}^{n} \times \mathbb{R}^{n} \times \mathbb{R}^{d}, \mathbb{R}^{n}\right)$ and $\lambda \in \mathbb{R}^{d}$ is an uncertain parameter;
ii) $(T x)(t)=\int_{t_{0}}^{t} g(t, s, x(s)) d s, g \in C\left(\mathbb{R}_{+} \times \mathbb{R}_{+} \times \mathbb{R}^{n}, \mathbb{R}^{n}\right)$;
iii) $t_{0}=\tau_{0}<\tau_{1}<\ldots<\tau_{k}<\ldots, \lim _{k \rightarrow \infty} \tau_{k}=\infty$;

\footnotetext{
*Received March 15, 2005; accepted for publication November 21, 2007.

†Department of Mathematics, Technical University - Sofia, Sliven, Bulgaria (gstamov@abv.bg).
} 
iv) $\Delta x\left(\tau_{k}\right)=x\left(\tau_{k}+0\right)-x\left(\tau_{k}-0\right), k=1,2, \ldots$;

v) $I_{k} \in C\left(\mathbb{R}^{n} \times \mathbb{R}^{d}, \mathbb{R}^{n}\right), k=1,2, \ldots$

We denote by $x(t)=x\left(t ; t_{0}, x_{0}\right)$ the solution of (1) with the initial condition $x\left(t_{0}+0 ; t_{0}, x_{0}\right)=x_{0}$.

Recall [2] the solution $x(t)$ is piecewise continuous function with points of discontinuity at the moments $\tau_{k}, k=1,2, \ldots$ at which it is continuous from the left.

Consider the following sets

$K=\left\{a \in C\left(\mathbb{R}_{+}, \mathbb{R}_{+}\right):\right.$a is monotone increasing in $\mathbb{R}_{+}$, and $\left.a(0)=0\right\}$.

$P C\left(\mathbb{R}_{+}, \mathbb{R}^{n}\right)=\left\{x: \mathbb{R}_{+} \rightarrow \mathbb{R}^{n}, x\right.$ is piecewise continuous function with

points of discontinuity of the first kind $\tau_{k}, k=1,2, \ldots$ and $\left.x\left(\tau_{k}-0\right)=x\left(\tau_{k}\right)\right\}$,

$G_{k}=\left\{(t, x) \in \mathbb{R}_{+} \times \mathbb{R}^{n}: \tau_{k}<t \leq \tau_{k+1}\right\}, k=0,1,2, \ldots$,

$W_{k}=\left\{(t, u) \in \mathbb{R}_{+}^{2}: \tau_{k}<t \leq \tau_{k+1}\right\}, k=0,1,2, \ldots$,

$V_{0}=\left\{V: \mathbb{R}_{+} \times \mathbb{R}^{n} \rightarrow \mathbb{R}_{+}\right.$, continuous on $G_{k}, V(t, 0)=0$, locally Lipschitz in $x$

and $\left.\lim _{\substack{t, x) \rightarrow\left(\tau_{k}, x_{0}\right) \\(t, x) \in G_{k+1}}} V(t, x)=V\left(\tau_{k}+0, x_{0}\right)\right\}$.

Definition 1. Let $V \in V_{0}$. For $(t, x) \in \cup_{k=0}^{\infty} G_{k}$ the upper right derivative $V(t, x)$ with respect to the impulsive differential system (1) is defined as

$$
D^{+} V(t, x(t))=\lim _{\delta \rightarrow 0+} i n f \delta^{-1}\{V(t+\delta, x(t)+\delta f(t, x(t),(T x)(t), \lambda))-V(t, x(t))\} .
$$

Our aim is to reduce the study of the system (1) to the study of a simple scalar impulsive differential equation with impulses at fixed moments and uncertain parameter.

For convenience let us state the following hypothesis.

$\left(A_{0}\right) w_{0}, w: \mathbb{R}_{+}^{3} \rightarrow \mathbb{R}_{+}$are continuous on $\left(\tau_{k}, \tau_{k+1}\right] \times \mathbb{R}_{+}^{2}$, there exist and are finite the limits

$$
w\left(\tau_{k}+0, u_{0}, \mu\right)=\lim _{\substack{(t, u, \mu) \rightarrow\left(\tau_{k}, u_{0}, \mu\right) \\(t, u) \in W_{k+1}}} w(t, u, \mu), w_{0}\left(\tau_{k}+0, u_{0}, \mu\right)=\lim _{\substack{(t, u, \mu) \rightarrow\left(\tau_{k}, u_{0}, \mu\right) \\(t, u) \in W_{k+1}}} w_{0}(t, u, \mu),
$$

$w_{0}(t, u, \mu) \leq w(t, u, \mu), h\left(t ; t_{0}, u_{0}\right)$ is the right maximal solution of the impulsive differential equation

$$
\left\{\begin{array}{l}
\dot{u}=w(t, u, \mu), t \neq \tau_{k}, t>t_{0}, \\
\Delta u\left(\tau_{k}\right)=u\left(\tau_{k}+0\right)-u\left(\tau_{k}-0\right)=\psi_{k}\left(u\left(\tau_{k}\right), \mu\right), k=1,2, \ldots, \\
u\left(t_{0}+0\right)=u_{0}, t_{0} \in \mathbb{R}_{+},
\end{array}\right.
$$

existing on $\left[t_{0}, \infty\right)$ and $\eta\left(t, t^{0}, v_{0}\right)$ is the left maximal solution of

$$
\left\{\begin{array}{l}
\dot{v}=w_{0}(t, v, \mu), t \neq \tau_{k}, t>t_{0}, \\
\Delta v\left(\tau_{k}\right)=v\left(\tau_{k}+0\right)-v\left(\tau_{k}-0\right)=\psi_{k}^{0}\left(v\left(\tau_{k}\right), \mu\right), k=1,2, \ldots, \\
v\left(t^{0}\right)=v_{0} \geq 0
\end{array}\right.
$$

existing on $t_{0} \leq t \leq t^{0}, \psi_{k}, \psi_{k}^{0} \in C\left(\mathbb{R}_{+}^{2}, \mathbb{R}\right), \psi_{k}(u, \mu), \psi_{k}^{0}(v, \mu), k=0,1,2, \ldots$ are nondecreasing in $u$ for $\mu \in \mathbb{R}_{+}$, and $\tau_{k}<\tau_{k+1}, \lim _{k \rightarrow \infty} \tau_{k}=\infty$. 
$\left(A_{1}\right) V \in V_{0}$ and for $t>t_{0}, x \in E_{0}$

$$
\begin{gathered}
D^{+} V(t, x(t)) \leq w(t, V(t, x(t)), \mu), t \neq \tau_{k}, \\
\left(D^{+} V(t, x(t)) \geq w(t, V(t, x(t)), \mu), t \neq \tau_{k}\right), k=0,1,2, \ldots
\end{gathered}
$$

where

$$
E_{0}=\left\{x \in P C\left[\mathbb{R}_{+}, \mathbb{R}^{n}\right]: V(s, x(s)) \leq \eta(s, t, V(t, x(t))), t_{0} \leq s \leq t\right\}
$$

and

$$
\begin{gathered}
V\left(t, x(t)+I_{k}(x(t), \lambda)\right) \leq \psi_{k}(V(t, x(t)), \mu), t=\tau_{k} \\
\left(V\left(t, x(t)+I_{k}(x(t), \lambda)\right) \geq \psi_{k}(V(t, x(t)), \mu),\right), t=\tau_{k}, k=1,2, \ldots .
\end{gathered}
$$

Theorem 1. Assume that conditions $(A)$ are hold.

Then, if $x(t)=x\left(t ; t_{0}, x_{0}\right)$ is any solution of (1) existing on $\left[t_{0}, \infty\right)$, we have $V(t, x(t)) \leq h\left(t ; t_{0}, u_{0}\right), t \geq t_{0}$, provided $V\left(t_{0}+0, x_{0}\right) \leq u_{0}$

or

$$
V(t, x(t)) \geq h\left(t ; t_{0}, u_{0}\right), t \geq t_{0}, \text { provided } V\left(t_{0}+0, x_{0}\right) \geq u_{0} .
$$

Proof. The proof of Theorem 1 is analogous of Theorem 3.8.1. in [3].

Now we consider the following definitions with respect to moving invariant manifolds of the systems (1) and (2).

Definition 2. Let $r_{k}=r_{k}(\lambda)>0, k=0,1,2, \ldots$. Then we say that the manifold $\Omega$, where

$$
\Omega=\cup_{k=1}^{\infty} \Omega_{k}, \Omega_{k}=\left\{x \in \mathbb{R}^{n}:(t, x) \in G_{k},|x|=r_{k}\right\}, k=0,1,2, \ldots
$$

is invariant and is uniformly asymptotically stable (UAS) with respect to (1), if

i) $\left|x_{0}\right|=r_{0} \Rightarrow|x(t)|=r_{k}, t \in\left(\tau_{k}, \tau_{k+1}\right], k=0,1,2, \ldots$,

ii) for given $\varepsilon>0$ and $t_{0} \in \mathbb{R}_{+}$

(a) there exists $\delta=\delta(\varepsilon)>0$ such that

$$
r_{0}-\delta<\left|x_{0}\right|<r_{0}+\delta \Rightarrow r_{k}-\varepsilon<|x(t)|<r_{k}+\varepsilon, t \in\left(\tau_{k}, \tau_{k+1}\right], k=0,1,2, \ldots,
$$

(b) there exist $\delta_{0}>0$ and $T=T(\varepsilon)>0$ such that if $t_{0}+T \in\left(\tau_{l}, \tau_{l+1}\right]$ for some $l=0,1,2, \ldots$ then

$$
r_{0}-\delta<\left|x_{0}\right|<r_{0}+\delta \Rightarrow r_{l}-\varepsilon<|x(t)|<r_{l}+\varepsilon, t \in\left(t_{0}+T, \tau_{l+1}\right]
$$

and

$$
r_{k}-\varepsilon<|x(t)|<r_{k}+\varepsilon, t \in\left(\tau_{k}, \tau_{k+1}\right], k \leq l+1,
$$


if $t_{0}+T=\tau_{p}+0$ for some $p=1,2, \ldots$, then

$$
r_{0}-\delta<\left|x_{0}\right|<r_{0}+\delta \Rightarrow r_{k}-\varepsilon<|x(t)|<r_{k}+\varepsilon, t \in\left(\tau_{k}, \tau_{k+1}\right], k \geq p,
$$

where $x(t)=x\left(t ; t_{0}, x_{0}\right)$ is solution of $(1)$.

Definition 3. Let $R_{k}=R_{k}(\mu)>0, k=0,1,2, \ldots$. Then we say that the manifold $u$

$$
u=\cup_{k=1}^{\infty} u_{k}, u_{k}=\left\{u \in \mathbb{R}_{+}:(t, u) \in W_{k}, u=R_{k}\right\}, k=0,1,2, \ldots
$$

is invariant and is uniformly asymptotically stable (UAS) with respect to (2) if

i) $u_{0}=R_{0} \Rightarrow R_{k}=u(t), t \in\left(\tau_{k}, \tau_{k+1}\right], k=0,1,2, \ldots$,

ii) for given $\varepsilon>0$ and $t_{0} \in \mathbb{R}_{+}$

(a) there exists $\delta=\delta(\varepsilon)>0$ such that

$$
R_{0}-\delta<u_{0}<R_{0}+\delta \Rightarrow R_{k}-\varepsilon<u(t)<R_{k}+\varepsilon, t \in\left(\tau_{k}, \tau_{k+1}\right], k=0,1,2, \ldots ;
$$

(b) there exist $\delta_{0}>0$ and $T=T(\varepsilon)>0$ such that if $t_{0}+T \in\left(\tau_{l}, \tau_{l+1}\right]$ for some $l=0,1,2, \ldots$ then

$$
R_{0}-\delta<u_{0}<R_{0}+\delta \Rightarrow R_{l}-\varepsilon<u(t)<R_{l}+\varepsilon, t \in\left(t_{0}+T, \tau_{l+1}\right],
$$

and

$$
R_{k}-\varepsilon<u(t)<R_{k}+\varepsilon, t \in\left(\tau_{k}, \tau_{k+1}\right], k \geq l+1
$$

if $t_{0}+T=\tau_{p}+0$ for some $p=1,2, \ldots$, then

$$
R_{0}-\delta<u_{0}<R_{0}+\delta \Rightarrow R_{k}-\varepsilon<u(t)<R_{k}+\varepsilon, t \in\left(\tau_{k}, \tau_{k+1}\right], k \geq p,
$$

where $u(t)=u\left(t ; t_{0}, u_{0}\right)$ is the solution of $(2)$.

We denote, for simplicity the following sets

$$
\begin{aligned}
& E_{1}^{(k)}=\left\{x ; x \in E_{0}, x(t) \in \mathbb{R}^{n} \backslash B_{r_{k}}\right\}, k=0,1,2, \ldots, \\
& E_{2}^{(k)}=\left\{x ; x \in E_{0}, x(t) \in B_{r_{k}} \cup S_{r_{k}}\right\}, k=0,1,2, \ldots .
\end{aligned}
$$

\section{Main results.}

Theorem 2. Assume that:

$\left(H_{0}\right)$ For each $\lambda \in \mathbb{R}^{d}$ there exist a sequences $\left\{r_{k}\right\}_{k=1}^{\infty}, r_{k}=r_{k}(\lambda)$ such that $r_{k}(\lambda)>0$ and $r_{k}(\lambda) \rightarrow 0$ as $|\lambda| \rightarrow 0, r_{k}(\lambda) \rightarrow \infty$ as $|\lambda| \rightarrow \infty$ for each $k=0,1,2, \ldots$.

$\left(H_{1}\right)$ There exist functions $V \in V_{0}$ and $a, b \in K$ such that

$$
b(|x|) \leq V(t, x) \text { for } t \neq \tau_{k}, x \in E_{1}^{(k)}
$$


and

$$
V(t, x) \leq a(|x|) \text { for } t \neq \tau_{k}, x \in E_{2}^{(k)}, k=0,1,2, \ldots
$$

$\left(H_{2}\right)$

$$
D^{+} V(t, x) \leq w\left(t, V(t, x), r_{k}\right) \text { for } t \neq \tau_{k}, x \in E_{1}^{(k)}
$$

and

$$
D^{+} V(t, x) \geq w\left(t, V(t, x), r_{k}\right) \text { for } t \neq \tau_{k}, x \in E_{2}^{(k)}, k=0,1,2, \ldots
$$

$\left(H_{3}\right)$

$$
V\left(\tau_{k}+0, x+I_{k}(x, \lambda)\right) \leq \psi_{k}(V(t, x), \mu), \text { for } x \in E_{1}^{(k)}
$$

and

$$
V\left(\tau_{k}+0, x+I_{k}(x, \lambda)\right) \geq \psi_{k}(V(t, x), \mu) \text { for } x \in E_{2}^{(k)}, k=0,1,2, \ldots
$$

$\left(H_{4}\right)$ For each sequence $\left\{r_{k}\right\}_{k=0}^{\infty}, r_{k}=r_{k}(\lambda)>0$ there exists a sequence $\left\{R_{k}\right\}_{k=0}^{\infty}$ such that $R_{k}=R\left(r_{k}\right) \geq 0$ such that $R_{k} \rightarrow 0$ as $r_{k} \rightarrow 0$ and $R_{k} \rightarrow$ $\infty$ as $r_{k} \rightarrow \infty, k=0,1,2, \ldots$ and $u=R, R=\cup_{k=0}^{\infty} R_{k}$ is invariant and $U A S$ relative to (2). Then if for any $r_{k}>0, a\left(r_{k}\right)=b\left(r_{k}\right)=$ $R\left(r_{k}\right)$, the manifold $\Omega=\cup_{k=1}^{\infty} \Omega_{k}$ is invariant and is $(U A S)$ relative to $(1)$.

Proof. Assume that condition $\left(H_{4}\right)$ be fulfilled for some $\left\{r_{k}\right\}_{k=0}^{\infty}, r_{k}=r_{k}(\lambda)>0$. First we shall prove that the manifold $\Omega$ is invariant with respect to (1).

If not there would exists a solution of (1) with $\left|x_{0}\right|=r_{0}$ and $t_{2}>t_{1} \geq t_{0}$ such that either

i) if $t_{1} \in\left(\tau_{k}, \tau_{k+1}\right]$ and $t_{2} \in\left(\tau_{l}, \tau_{l+1}\right], k \geq l$, then $\left|x\left(t_{1}\right)\right|=r_{k},\left|x\left(t_{2}\right)\right|>r_{l}, x \in E_{0}$ such that $x(t) \in \mathbb{R}^{n} \backslash B_{r_{\sigma}}, t \in\left[t_{1}, t_{2}\right]$, where $\sigma=k$ as $l=k$, or $\sigma=k, k+1, \ldots, l$ as $l>k$.

From $\left(H_{1}\right)$ and $\left(H_{2}\right)$ for $V(t, x(t))$ it follows that

$$
\begin{gathered}
D^{+} V(t, x(t)) \leq w\left(t, u\left(t ; t_{1}, V\left(t_{1}, x\left(t_{1}\right)\right)\right), r_{\sigma}\right) \text { if } t \in\left[t_{1}, t_{2}\right] \backslash\left\{\tau_{\sigma} \in\left[t_{1}, t_{2}\right]\right\} \\
V\left(\tau_{\sigma}+0, x\left(\tau_{\sigma}\right)+I_{\sigma}\left(x\left(\tau_{\sigma}\right), \lambda\right)\right) \leq \psi_{\sigma}\left(V\left(\tau_{\sigma}, x\left(\tau_{\sigma}\right)\right), r_{\sigma}\right) \text { for } \tau_{\sigma} \in\left[t_{1}, t_{2}\right]
\end{gathered}
$$

or

ii) if $t_{1} \in\left(\tau_{k}, \tau_{k+1}\right]$ and $t_{2} \in\left(\tau_{l}, \tau_{l+1}\right], k \geq l,\left|x\left(t_{1}\right)\right|=r_{k},\left|x\left(t_{2}\right)\right|<r_{l}, x \in E_{0}$ such that $x(t) \in B_{r_{\sigma}} \cup S_{r_{\sigma}}, t \in\left[t_{1}, t_{2}\right]$, where $\sigma=k$ as $l=k$, and $\sigma=k, k+1, \ldots, l$ as $l>k$.

From $\left(H_{1}\right)$ and $\left(H_{2}\right)$ it follows that

$$
\begin{gathered}
D^{+} V(t, x(t)) \geq w\left(t, u\left(t: t_{1}, V\left(t_{1}, x\left(t_{1}\right)\right)\right), r_{\sigma}\right) \text { if } t \in\left[t_{1}, t_{2}\right] \backslash\left\{\tau_{\sigma} \in\left[t_{1}, t_{2}\right]\right\}, \\
V\left(\tau_{\sigma}+0, x\left(\tau_{\sigma}\right)+I_{\sigma}\left(x\left(\tau_{\sigma}\right), \lambda\right)\right) \geq \psi_{\sigma}\left(V\left(\tau_{\sigma}, x\left(\tau_{\sigma}\right)\right), r_{\sigma}\right) \text { for } \tau_{\sigma} \in\left[t_{1}, t_{2}\right],
\end{gathered}
$$

where $u\left(t, t_{1}, V\left(t_{1}, x\left(t_{1}\right)\right)\right)$ is the solution of (2) through $\left(t_{1}, V\left(t_{1}, x\left(t_{1}\right)\right)\right)$. 
Using comparison Theorem 1 in case (i) we have

$$
V(t, x(t)) \leq u\left(t ; t_{1}, V\left(t_{1}, x\left(t_{1}\right)\right)\right), t_{1} \leq t \leq t_{2},
$$

or in case ii)

$$
V(t, x(t)) \geq u\left(t ; t_{1}, V\left(t_{1}, x\left(t_{1}\right)\right)\right), t_{1} \leq t \leq t_{2} .
$$

Hence in i) we obtain

$$
\begin{aligned}
& b\left(r_{\sigma}\right)<b\left(\left|x\left(t_{2}\right)\right|\right) \leq V\left(t_{2}, x\left(t_{2}\right)\right) \leq u\left(t_{2} ; t_{1}, a\left(\left|x\left(t_{1}\right)\right|\right)\right)= \\
= & u\left(t_{2} ; t_{1}, a\left(r_{\sigma}\right)\right)=b\left(r_{\sigma}\right)=a\left(r_{\sigma}=R_{\sigma}\right), \sigma=k, k+1, \ldots, l,
\end{aligned}
$$

which is a contradiction.

In case ii) we obtain

$$
\begin{aligned}
& a\left(r_{\sigma}\right)>a\left(\left|x\left(t_{2}\right)\right|\right) \geq V\left(t_{2}, x\left(t_{2}\right)\right) \geq u\left(t_{2}, t_{1}, b\left(\left|x\left(t_{1}\right)\right|\right)\right)= \\
= & u\left(t_{2}, t_{1}, b\left(r_{\sigma}\right)\right)=b\left(r_{\sigma}\right)=a\left(r_{\sigma}\right)=R_{\sigma}, \sigma=k, k+1, \ldots, l,
\end{aligned}
$$

which also is a contradiction.

Let $\varepsilon>0$ and $t_{0} \in \mathbb{R}_{+}$be given. Suppose that $u=R$ is $U S$. Then since $a\left(r_{k}\right)=$ $b\left(r_{k}\right)=R_{k}, k=1,2, \ldots$ given $a\left(r_{k}-\varepsilon\right), b\left(r_{k}+\varepsilon\right)$, there exist $\varepsilon_{1}>0, \delta_{1}>0, \delta>0$ such that

$$
R_{k}+\delta_{1}=a\left(r_{k}+\delta\right)<b\left(r_{k}+\varepsilon\right)=R_{k}+\varepsilon_{1}, k=0,1,2, \ldots
$$

and

$$
R_{k}-\varepsilon_{1}=a\left(r_{k}-\varepsilon\right)<b\left(r_{k}-\delta\right)=R_{k}-\delta_{1}, k=0,1,2, \ldots
$$

Satisfying $R_{0}-\delta_{1}<u_{0}<R_{0}+\delta_{1}$ implies $R_{k}-\varepsilon_{1}<u(t)<R_{k}+\varepsilon_{1}, t \geq t_{0}, k=$ $0,1,2, \ldots$ where $u(t)$ is solution of (2). We claim that with this $\delta>0$ the manifold $\Omega$ is $U S$, that is

$$
r_{0}-\delta<\left|x_{0}\right|<r_{0}+\delta \Rightarrow r_{k}-\varepsilon<|x(t)|<r_{k}+\varepsilon, t \geq t_{0}, k=1,2, \ldots .
$$

If this is not true, there would exist a solution $x(t)$ of (1) with $r_{0}-\delta<\left|x_{0}\right|<r_{0}+\delta$ and $t_{2}>t>t_{1}$ such that either

(a) $\left|x\left(t_{2}\right)\right|=r_{l}+\varepsilon,\left|x\left(t_{1}\right)\right|=r_{k}+\delta$ and $x \in E_{0}$ such that $x(t) \in \mathbb{R}^{n} \backslash\left(B_{r_{\sigma}} \cup S_{r_{\sigma}}\right), t \in$ $\left[t_{1}, t_{2}\right], t_{1} \in\left(\tau_{k}, \tau_{k+1}\right], t_{2} \in\left(\tau_{l}, \tau_{l+1}\right], l \geq k, \sigma=k, k+1, \ldots, l$.

or

(b) $\left|x\left(t_{2}\right)\right|=r_{l}-\varepsilon,\left|x\left(t_{1}\right)\right|=r_{k}-\delta$ and $x \in E_{0}$ such that $x(t) \in B_{r_{\sigma}}, t \in\left[t_{1}, t_{2}\right]$, $t_{1} \in\left(\tau_{k}, \tau_{k+1}\right], t_{2} \in\left(\tau_{l}, \tau_{l+1}\right], l \geq k, \sigma=k, k+1, \ldots, l$.

Consider (a). As before, we have

$$
V(t, x(t)) \leq u\left(t ; t_{1}, V\left(t_{1}, x\left(t_{1}\right)\right)\right), t \in\left[t_{1}, t_{2}\right]
$$


and therefore, we arrive at the contradiction

$b\left(r_{\sigma}+\varepsilon\right)=b\left(\left|x\left(t_{2}\right)\right|\right) \leq V\left(t_{2}, x\left(t_{2}\right)\right) \leq u\left(t_{2} ; t_{1}, a\left(r_{\sigma}+\delta\right)\right)<b\left(r_{\sigma}+\varepsilon\right), \sigma=k, k+1, \ldots, l$.

Similarly, in case (b) we first get

$$
V(t, x(t)) \geq u\left(t, t_{1}, V\left(t_{1}, x\left(t_{1}\right)\right)\right), t \in\left[t_{1}, t_{2}\right],
$$

and then it follows that

$a\left(r_{\sigma}-\varepsilon\right)=a\left(\left|x\left(t_{2}\right)\right|\right) \geq V\left(t_{2}, x\left(t_{2}\right)\right) \geq u\left(t_{2} ; t_{1}, a\left(r_{\sigma}-\delta\right)\right)>a\left(\rho_{\sigma}-\varepsilon\right), \sigma=k, k+1, \ldots, l$

which is a contradiction. Hence $\Omega$ is $U S$.

To prove $U A S$ of the set $\Omega$ let us first fix $\varepsilon_{k}=r_{k}, k=1,2, \ldots$ and designate by $\delta_{k}=\delta\left(r_{k}\right)$ so that we obtain

$$
b\left(r_{k}-\delta_{k}\right)<u_{0}<a\left(r_{k}+\delta\right) \Rightarrow 0<u(t)<b\left(2 r_{k}\right), t \geq t_{0}, k=0,1,2, \ldots,
$$

and

$$
r_{0}-\delta_{0}<\left|x_{0}\right|<r_{0}+\delta_{0} \Rightarrow 0<|x(t)|<2 r_{k}, t \geq t_{0}, k=0,1,2, \ldots
$$

Assume that $u=R$ is $U A S$ and let $\delta=\delta(\varepsilon)$ be the same number corresponding to $\varepsilon$ is $U S$. Then given $b\left(r_{k}+\delta\right), a\left(r_{k}-\delta\right)$, there exists $T=T(\varepsilon)>0$ such that

iii) if $t_{0}+T \in\left(\tau_{l}, \tau_{l+1}\right]$ for some $l=1,2, \ldots$ then from

$$
b\left(r_{0}-\delta_{0}\right)<u_{0}<a\left(r_{0}+\delta_{0}\right) \Rightarrow a\left(r_{l}-\delta\right)<u(t)<b\left(r_{l}+\delta\right), t \in\left(t_{0}+T, \tau_{l+1}\right]
$$

and

$$
a\left(r_{k}-\delta\right)<u(t)<b\left(r_{k}+\delta\right), t \in\left(\tau_{k}, \tau_{k+1}\right], k \geq l+1,
$$

iv) if $t_{0}+T=\tau_{p}$ for some $p=1,2, \ldots$ then from

$$
b\left(r_{0}-\delta_{0}\right)<u_{0}<a\left(r_{0}+\delta_{0}\right) \Rightarrow a\left(r_{k}-\delta\right)<u(t)<b\left(r_{k}+\delta\right), t \in\left(\tau_{k}, \tau_{k+1}\right], k \geq p .
$$

Since $\Omega$ is $U S$ it is enough to show that there exists $t^{*} \in\left(\tau_{q}, \tau_{q+1}\right] \subset\left(t_{0}, t_{0}+T\right)$ satisfying $r_{q}-\delta<\left|x\left(t^{*}\right)\right|<r_{q}+\delta$. If $t^{*}$ not exists, then for $t_{0}+T \in\left(\tau_{l}, \tau_{l+1}\right]$ we have either

(a) $x \in E_{0}$ such that $x(t) \in \mathbb{R}^{n} \backslash B_{r_{\sigma}+\delta}$ for all $t \in\left[t_{0}, t_{0}+T\right] \backslash\left\{\tau_{\sigma} \in\left(t_{0}, t_{0}+T\right], \sigma=\right.$ $1,2, \ldots, l\}$

or

(b) $x \in E_{0}$ such that $x(t) \in B_{r_{\sigma}+\delta} \cup S_{r_{\sigma}+\delta}$ for all $t \in\left[t_{0}, t_{0}+T\right] \backslash\left\{\tau_{\sigma} \in\right.$ $\left.\left(t_{0}, t_{0}+T\right], \sigma=1,2, \ldots, l\right\}$.

Then we get relative to (a)

$$
b\left(r_{\sigma}+\delta\right) \leq V\left(t_{0}+T, x\left(t_{0}+T\right)\right) \leq u\left(t_{0}+T ; t_{0}, a\left(r_{\sigma}+\delta_{0}\right)\right)<b\left(r_{\sigma}+\delta\right),
$$

for $\sigma=0,1,2, \ldots, l$ which is contraction. Similarly, in case (b), it follows that

$$
a\left(r_{\sigma}-\delta\right) \geq V\left(t_{0}+T, x\left(t_{0}+T\right)\right) \geq u\left(t_{0}+T ; t_{0}, b\left(r_{\sigma}-\delta_{0}\right)\right)>a\left(r_{\sigma}-\delta\right),
$$


for $\sigma=1,2, \ldots, l$ which is again a contraction. Hence there exists $t^{*} \in\left[t_{0}, t_{0}+T\right]$ satisfying $r_{q}-\delta<\left|x\left(t^{*}\right)\right|<r_{q}+\delta$ and the proof of Theorem 2 is complete.

Remark 1. We denote that the main results in the paper follows from the estimate of Lyapunov's functions on the minimal class $E_{0}$ of assumption $\left(A_{1}\right)$. This class depends on the choice of the functions $w_{0}(t, v, \mu)$, and $\psi_{k}^{0}(v, \mu), k=1,2, \ldots$ and the special cases of these choices are considered in [3,4].

\section{REFERENCES}

[1] Bainov D., Kostadinov S., NGuyen Van Min, Dichotomies and Integral Manifolds of Impulsive Differential Equations, SCT Publishing, Singapore, 1994.

[2] Bainov D., Simeonov P.S., Stability of the Solutions of a System of Integro-Differential Equations with Impulse Effect, Math. Rep. Toyama Univ., Vol. 9 (1986), 1-24.

[3] Lakshmikantham, V., Leela, S. and Martynyuk, A. A., Stability Analysis of Nonlinear Systems, Marcel Dekker, New York 1989.

[4] Lakshmikantham, V., Leela, S. and Martynyuk, A. A., Practical Stability of Nonlinear Systems, World Scientific Publishing, Singapore 1990.

[5] Lakshmikantham V. and Vatsala A. S. Stability of Moving Invariant Sets, Stability and Control: Theory, Methods and Applications, 5 (1997), 79-83.

[6] Shendge, G. R. A new approach to the stability theory of functional differential equations, J. Math. Anal. Apll., Vol. 95, (1983), 319-334.

[7] Siljak, D. D., Ikeda, M. And Ohta, Y. Parametic Stability, Proccedings Universita di GenovaOhio State University Joint Conference: Birkhauser, (1991), 1-20.

[8] Stamov, G. Second Method of Lyapunov for Existence of Almost Periodic Solutions for Impulsive Integro-Differential Equations, KYUNGPOOK Math. J., Vol. 43 (2003), 221-231.

[9] Rama Mohana Rao, M. Raghavendra V., Asymptotic Stability Properties of Volterra Integro-Differential Equations, Nonlinear Analysis, T. M. A., Vol. 11, (1987), 475-480.

[10] Vatsala A. S. and Deo S. G. Stability of Moving Invariant Sets for Functional Differential Systems, Int. J. of Nonl. Diff. Eq.: Theory, Methods and Applications, 3 (1997), 179-186. 